\title{
Force-Dependent Mechanical Unfolding Pathways of GFP
}

\author{
Penghui Cao ${ }^{\mathrm{a}, \mathrm{b}}$, Weiwei Tao ${ }^{\mathrm{a}}$, Harold S. Park ${ }^{\mathrm{a}, *}$ \\ ${ }^{a}$ Department of Mechanical Engineering, Boston University, Boston, MA 02215 \\ ${ }^{b}$ Department of Nuclear Science and Engineering, Massachusetts Institute of Technology, \\ Cambridge MA 02139
}

\begin{abstract}
We characterize the force-dependent unfolding pathways and intermediate configurations of the green fluorescence protein (GFP) using novel atomistic simulations based on potential energy surface exploration. By using this approach, we are able to unfold GFP to significantly longer end-to-end distances, i.e. $40 \mathrm{~nm}$, as compared to that seen in previous atomistic simulation studies. We find that there are four intermediate states between 5 and $40 \mathrm{~nm}$ end-to-end distance, where the intermediate configurations and unfolding pathways are strongly force-dependent. We additionally calculate the force-dependent lifetime of the $14 \mathrm{~nm} \alpha \beta_{1}$ intermediate, and demonstrate that it obeys Bell's formula.
\end{abstract}

Keywords: gfp, force clamping, intermediate configuration

\section{Introduction}

Over the past decade, researchers have successfully exploited experimental advances in nanomechanical testing to mechanically unfold and refold individual proteins (Neuman and Nagy, 2008; Bustamante et al., 2004). One of the proteins that has been studied most frequently is the green fluorescence protein (GFP), which because it shows green fluorescence when subject to light at a specific wavelength, has been proposed to be used as an optical strain sensor (Zimmer, 2002).

\footnotetext{
*Corresponding author

Email addresses: pcao@mit.edu (Penghui Cao), parkhs@bu.edu (Harold S. Park)
} 
Because of its potential as a mechanically active biological sensor, the mechanical unfolding of GFP has been studied using both experiments (Dietz and Rief, 2004; Mickler et al., 2007; Perez-Jimenez et al., 2006) and computer simulations (Hyeon et al., 2006; Eyal and Bahar, 2008; Caraglio et al., 2011; Saeger et al., 2012), where a focus of the simulations has been to identify its various intermediate configurations. The simulations have used various forms of atomistic modeling and approximation, including steered molecular dynamics (SMD) (Saeger et al., 2012), an Ising-like model (Caraglio et al., 2011), and an elastic network model (Eyal and Bahar, 2008). However, due to the known time scale limitation in SMD, the longest simulation time has been on the order of 100 nanoseconds, with the largest end to end distance (ETED) of $14 \mathrm{~nm}$ (Saeger et al., 2012). Thus, while a complete characterization of the intermediate configurations and unfolding pathways of GFP is necessary to understand its capacity as a strain sensor, this information, particularly with regards to its unfolding pathways for ETEDs larger than $14 \mathrm{~nm}$, are still not clear.

In this work, we employ the self-learning metabasin escape (SLME) method (Kushima et al., 2009; Cao et al., 2012, 2013), which couples potential energy surface exploration with constant tensile forces ranging from $100 \mathrm{pN}$ to 300 $\mathrm{pN}$ to study the mechanical unfolding of GFP. We note that many previous works in utilizing potential energy surface exploration to study the unfolding dynamics and mechanisms of proteins have been performed. These works include the metadynamics approach of Marinelli et al. (2009), which has recently been used to study the dynamic behavior of protein molecules (Formoso et al., 2015; Berteotti et al., 2011; Laio and Parrinello, 2002), and the discrete path sampling (DPS) method of Wales (Wales, 2002, 2004), who used PES exploration to determine rate constants for different transition states, and the SLME method for ubiquitin (Cao et al., 2015) and prion (Tao et al., 2015).

Our key findings are associated with the unfolding of GFP well-beyond that seen in previous simulation studies, where we reach ETEDs of $40 \mathrm{~nm}$. In doing so, we find that there are three intermediate states between 14 and $40 \mathrm{~nm}$ ETED, where the intermediate configurations and unfolding pathways are strongly force-dependent. We additionally calculate the force-dependent lifetime of the $14 \mathrm{~nm} \alpha \beta_{1}$ intermediate, and demonstrate that it obeys Bell's formula. 


\section{Methods}

It is well-known that the SMD simulations for force-induced unfolding of proteins exhibit major drawbacks as compared to the corresponding force clamp experiments (Elber, 2005; Carrion-Vazquez et al., 2003; Li et al., 2010; Stirnemann et al., 2013). In particular, the time scales accessible by SMD are on the order of hundreds of nanoseconds, while the unfolding time of GFP is on the order of microseconds to milliseconds (Dietz and Rief, 2004). In order to study the mechanical unfolding of GFP at experimentally-relevant (100$300 \mathrm{pN}$ ) forces and longer time scales, we utilize the SLME method of Cao et al. (2012, 2013), which couples potential energy surface (PES) sampling with tensile deformation, and which was recently used to bring new insights into the mechanical unfolding pathways of the proteins ubiquitin (Cao et al., 2015) and prion (Tao et al., 2015).

The SLME method is an improved version of the original autonomous basin climbing (ABC) method of Kushima et al. (2009). This improvement was necessary due to the substantial increase in computational expense that occurs as PES exploration continues due to the need to store the penalty functions that are used to push the system out of local energy minima such that the system does not re-explore any energy basins. The ABC method has recently been used to probe extremely slow dynamical processes like diffusion in amorphous silicon (Yan et al., 2015), nanocrystal creep (Lau et al., 2010), void nucleation and growth (Fan et al., 2011) and dislocationdefect interactions at slow strain rates (Fan et al., 2013).

In the SLME approach, quartic penalty functions are utilized to push the system out of potential energy wells in which it can become stuck due to the relatively low forces (i.e. $100 \mathrm{~s}$ of $\mathrm{pN}$ for proteins) that are constantly applied. Upon application of a sufficient number of penalty functions, the system escapes over the lowest energy barrier to a neighboring potential energy well, where penalty functions are again applied if the applied force is not sufficient to lower the energy barrier to enable the system to escape. Thus, the penalty functions can be physically interpreted as thermal activation that assists the mechanical force in enabling the system to escape from a local energy minimum. This procedure is repeated until GFP is unfolded. In going through this procedure, the system is able to find and pass through the relevant intermediate configurations.

The unfolding time is estimated using transition state theory (Popa et al., 
2011; Hanggi et al., 1990) via

$$
\tau=\sum_{i=1}^{N}\left(\nu \exp ^{-Q_{i} / k_{B} T}\right)^{-1},
$$

where $Q_{i}$ is the energy barrier separating energy minima $i-1$ and $i, N$ is total number of energy minima explored on the unfolding path, $\nu$ is a frequency prefactor, $T$ is the temperature and $k_{B}$ is the Boltzmann constant. We used the value $\nu=1 e^{9} \mathrm{~s}^{-1}$ as was utilized in the experimental GFP studies of Dietz and Rief (2004).

We employed the AMBER99sb potential field which utilizes an implicit solvent model for water, while we used the protein with the Protein Data Bank (PDB) structure of $1 \mathrm{fgl}$ (Yang et al., 1996) for the native configuration of GFP. The native GFP structure was first equilibrated at $300 \mathrm{~K}$, and then energy minimization was performed to generate the corresponding local energy minimum. At both the $\mathrm{N}$ and C-termini, a constant pulling force ranging from 100 to $300 \mathrm{pN}$ was subsequently applied using the SLME method (Cao et al., 2013). All simulations were performed using the open source GROMACS simulation package (Gromacs, 2014; Hess et al., 2008).

To construct the free energy profile of GFP along an unfolding path, we performed umbrella sampling while accounting for the clamping force. We sampled every $1 \AA$ of end-to-end distance using a $1000 \mathrm{kJmol}^{-1} \AA^{-2}$ force constant for a 1 ns equilibration period. The potential of mean force (PMF) was then extracted by the Weighted Histogram Analysis Method (WHAM) (Hub et al., 2010).

\section{Results}

We conducted 50 independent SLME simulations at clamping forces ranging from 100 to $300 \mathrm{pN}$, with 10 simulations performed at each force level. The unfolding pathways for the different forces are shown in Fig. 1 where the plateau regions indicate intermediate states. These intermediate states are separated by energy barriers which must be crossed for additional unfolding to occur. For such small forces, SMD is not able to simulate large barrier crossing due to the well-known time scale limitation. However, by adding penalty functions, the SLME simulations are able to access a much longer time scale, and as shown in Fig. 1(c), is able to unfold GFP to a distance of $40 \mathrm{~nm}$, which far exceeds the $15 \mathrm{~nm}$ unfolding that was previously observed 

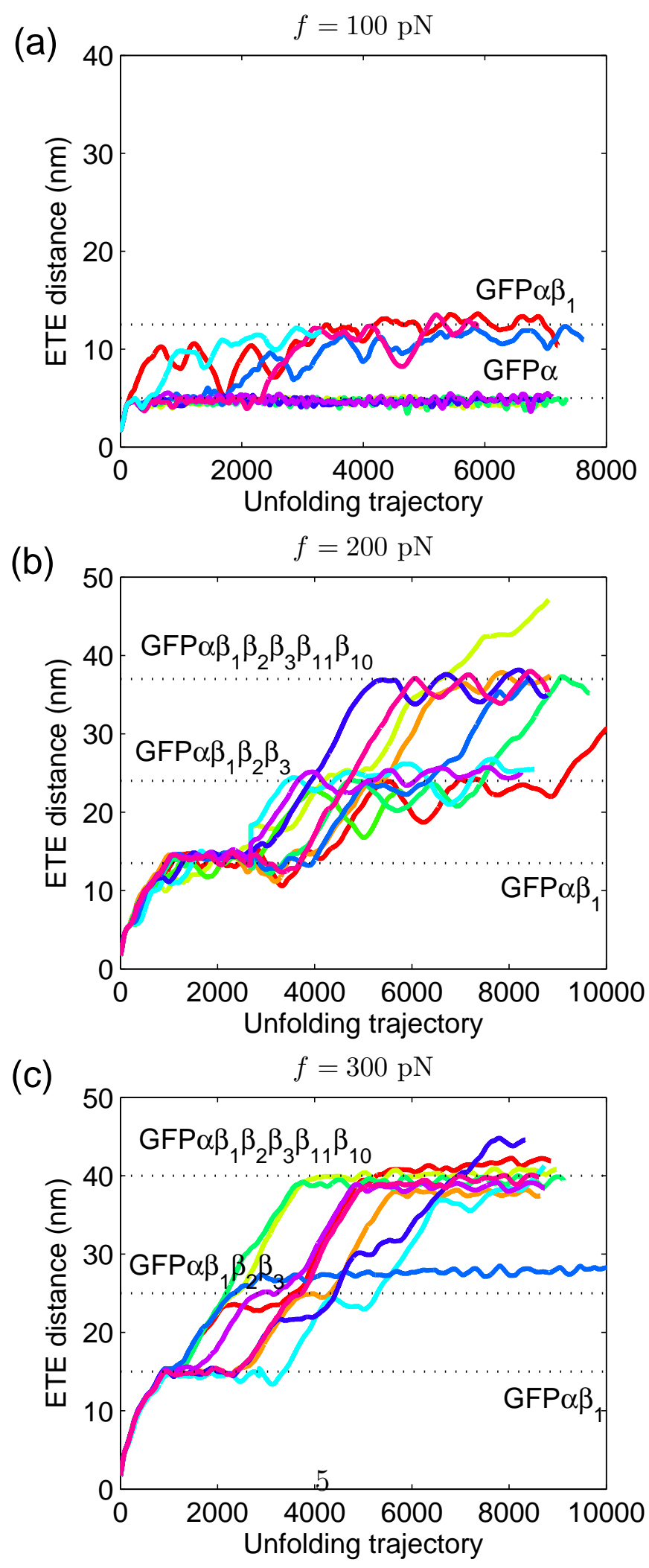

Figure 1: The mechanical unfolding of GFP obtained using the SLME method as shown through the end-to-end distance vs. local minima explored for unfolding at 100, 200 and $300 \mathrm{pN}$. Unfolding trajectory refers to the total number of local energy minima found for the SLME simulation. 
using SMD (Saeger et al., 2012). We note that while this $40 \mathrm{~nm}$ unfolding distance is larger than seen in previous SMD simulations, it is still shorter than the $76 \mathrm{~nm}$ distance that is needed to completely unfold GFP (Dietz and Rief, 2004). In agreement with previous SMD simulations (Saeger et al., 2012), at $100 \mathrm{pN}$ the corked state was observed at an ETED of $5 \mathrm{~nm}$ while the ripple state was observed at an ETED of $14 \mathrm{~nm}$. Larger unfolding distances for the $100 \mathrm{pN}$ clamping force were not achieved due to computational limitations.

In all constant force unfolding simulations for all force levels, the $\mathrm{N}$ terminal $\alpha$-helix unravels first from the barrel, which agrees with earlier constant velocity coarse grain model simulations (Mickler et al., 2007; Caraglio et al., 2011). However, the constant velocity simulations exhibit a bifurcation in the unfolding pathways after the unfolding of the $\alpha$-helix. Specifically, the $\alpha \rightarrow \beta_{1}$ pathway is observed in $72 \%$ of the simulations, while the $\alpha \rightarrow \beta_{11}$ pathway is observed in 28\% of the simulations (Mickler et al., 2007; Caraglio et al., 2011). Those values for each pathway can be changed by cross-link mutations, or by changing the clamping force direction.

Fig. 1 also demonstrates that the unfolding pathways depend on the magnitude of the clamping force. At $300 \mathrm{pN}, 90 \%$ of the unfolding trajectories take two unfolding steps to unfold to the ETED of $40 \mathrm{~nm}$, while in contrast $70 \%$ of the simulations follow 3-step unfolding at the smaller clamping force of $200 \mathrm{pN}$. For the $100 \mathrm{pN}$ simulations, we were unable to, due to computational limitations, unfold GFP past an ETED of $14 \mathrm{~nm}$.

We show in Fig. 2 two representative unfolding pathways for the $200 \mathrm{pN}$ clamping force. The pathway analyzed in Figs. 2(a)-(c) passes through one intermediate at $14 \mathrm{~nm}$, while the pathway analyzed in Figs. 2(d)-(f) passes through two intermediates, one at 14 and the other at $23 \mathrm{~nm}$. Figs. 2(b) and (e) show the heights of the energy barriers that are crossed over during the unfolding as a function of ETED. As can be seen, the pathway shown in Fig. 2(d) encounters many more large energy barriers starting around ETED in Fig. 2(e) as compared to the pathway shown in Fig. 2(b), which explains why it passes through an intermediate around ETED of $23 \mathrm{~nm}$. The second salient point is the unfolding time as a function of ETED in Figs. 2(c) and (f). In particular, the unfolding time from 0 to $38 \mathrm{~nm}$ ETED is on a time scale of tens of $\mu$ s, which is a time scale that is difficult to reach using classical MD without substantial parallel computing resources. In contrast, all of the computations performed in this work were done on a standard Linux desktop computer, which demonstrates the potential of the SLME method 

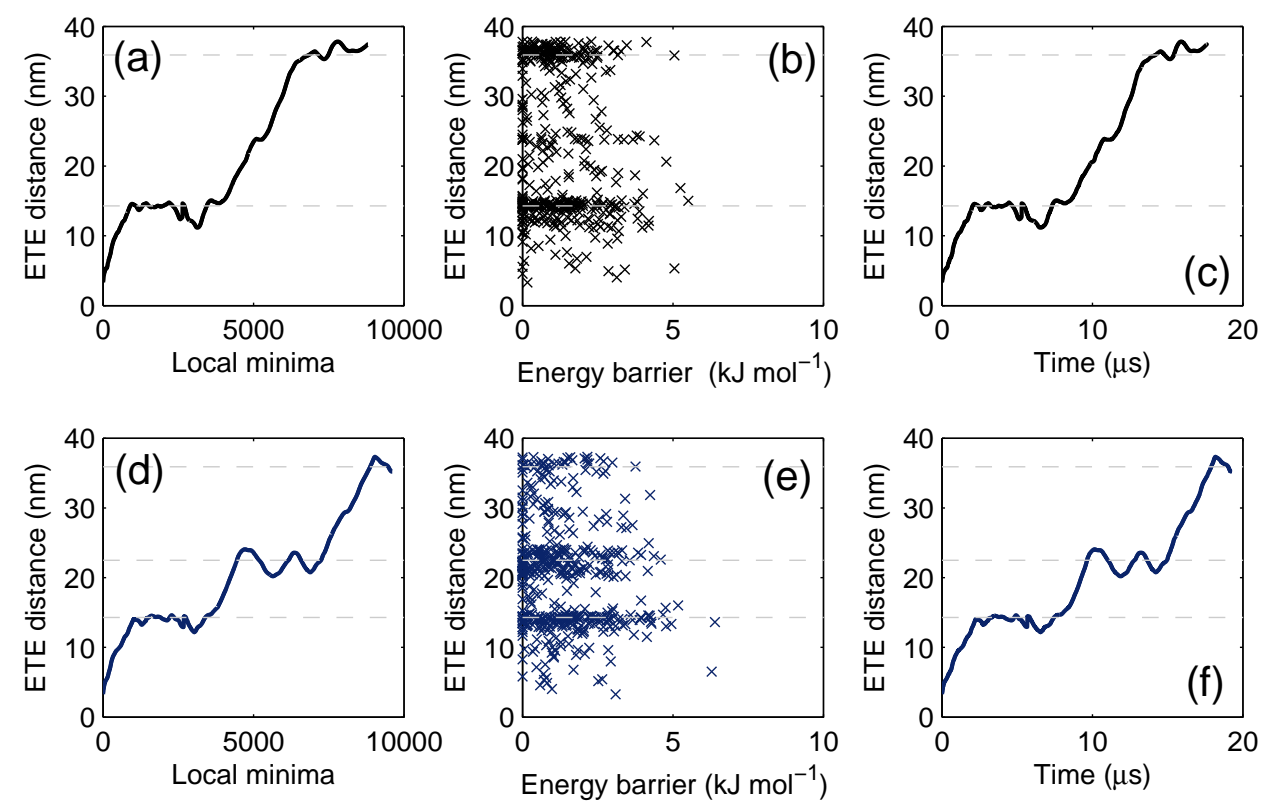

Figure 2: (a)(d) Two representative unfolding pathways at $200 \mathrm{pN}$. (b)(e) Distribution of energy barriers along each unfolding pathway. (c)(f) Unfolding time as a function of ETED. 
(a)

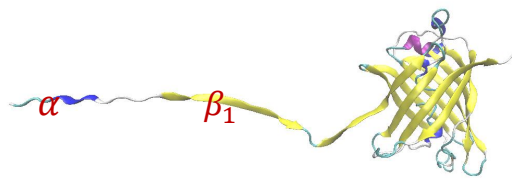

Intermediate $\alpha \beta_{1}$

(b)

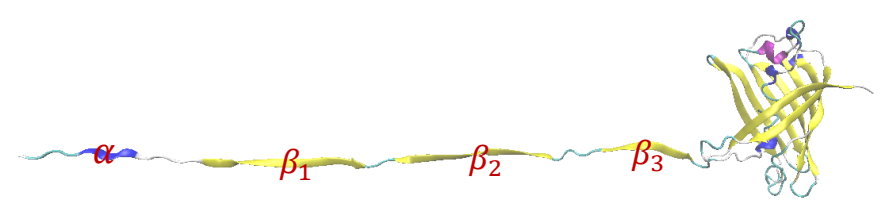

Intermediate $\alpha \beta_{1} \beta_{2} \beta_{3}$

(c)

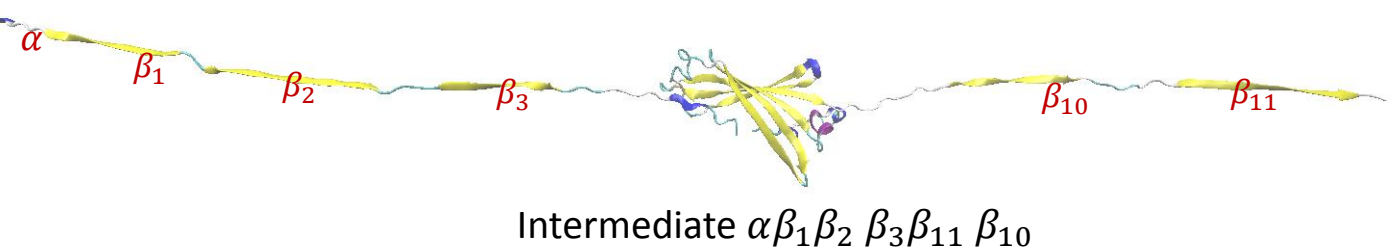

Figure 3: Intermediate states observed during the mechanical unfolding GFP at $200 \mathrm{pN}$. (A) $\alpha \beta_{1}$, (B) $\alpha \beta_{1} \beta_{2} \beta_{3}$, (C) $\alpha \beta_{1} \beta_{2} \beta_{3} \beta_{11} \beta_{10}$.

for studying the unfolding of proteins at experimentally-relevant times scales.

4 intermediate states, $\alpha, \alpha \beta_{1}, \alpha \beta_{1} \beta_{2} \beta_{3}$ and $\alpha \beta_{1} \beta_{2} \beta_{3} \beta_{11} \beta_{10}$ were observed in the SLME simulations. The $\alpha$ intermediate state is formed after the initial rupture of the N-terminal $\alpha$-helix. This intermediate is observed only in the $100 \mathrm{pN}$ pathways, and is not observed for larger clamping forces because the value of the clamping force required to unravel $\beta_{1}$ is around $100 \mathrm{pN}$ (Hyeon et al., 2006; Dietz and Rief, 2004). The unraveling of $\beta_{1}$ followed by $\alpha$ forms the intermediate $\alpha \beta_{1}$, or the ripped state (Saeger et al., 2012) as shown in Fig. $3(\mathrm{a})$, with a corresponding ETED for the $\alpha \beta_{1}$ intermediate state in the range of 12-14 nm. The unraveling of $\beta_{1}$ leaves $\beta_{2} \beta_{3}$ unshielded, and thus they begin unraveling and the length of the next intermediate, corresponding to $\alpha \beta_{1} \beta_{2} \beta_{3}$ in Fig. 3(b), occurs at an ETED of around $25 \mathrm{~nm}$. After passing through this intermediate, $\beta_{11} \beta_{10}$ unfolds resulting in a new intermediate state of 

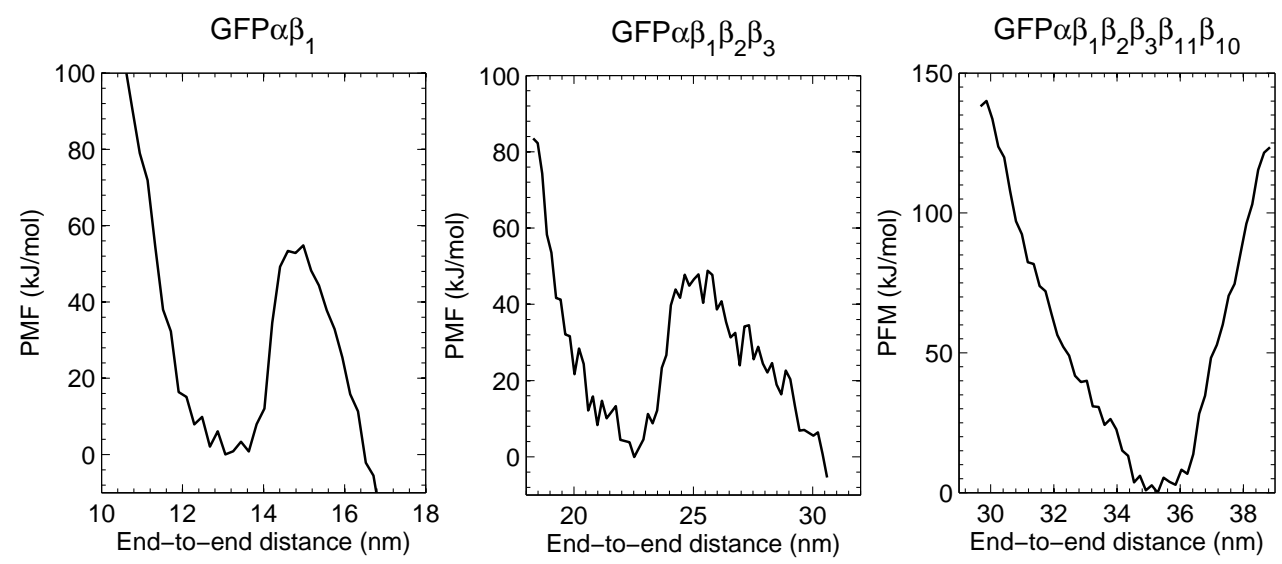

Figure 4: Free energy profile as a function of end-to-end distance for intermediate states found during the $200 \mathrm{pN}$ unfolding of GFP.

$\alpha \beta_{1} \beta_{2} \beta_{3} \beta_{11} \beta_{10}$ at an ETED of about $39 \mathrm{~nm}$ as shown in Fig. 3(c). These intermediates states correspond to the minima on the free energy profile, as we now discuss.

In Fig. 4, we report the PMF profile for a clamping force of $200 \mathrm{pN}$ applied during the umbrella sampling simulation. Three distinct local minima can be observed for ETEDs of about 13, 23 and $36 \mathrm{~nm}$. As previously discussed, these local minima correspond to the mechanical intermediate configurations of $\alpha \beta_{1}, \alpha \beta_{1} \beta_{2} \beta_{3}$ and $\alpha \beta_{1} \beta_{2} \beta_{3} \beta_{11} \beta_{10}$ shown in Fig. 3. Furthermore, the fact that these intermediate configurations correspond to local energy minima validates them as true intermediate states during the mechanical unfolding process. To compare with existing experimental data, we note that in the 2004 work of Dietz et al. Dietz and Rief (2004), the energy barrier for transition from GFP $\Delta \alpha$ to GFP $\Delta \alpha \Delta \beta$ was found to be about $23 k_{B} T$, or about $57 \mathrm{~kJ} / \mathrm{mol}$. Fig. 4(left) shows that the barrier for GFP $\Delta \alpha \Delta \beta$ is about $47 \mathrm{~kJ} / \mathrm{mol}$, which gives reasonably good agreement.

We now analyze the force-dependent dynamics of one particular intermediate configuration, the the $\alpha \beta_{1}$ intermediate. This intermediate state was chosen as it is observed at all clamping force levels we used for the SLME simulations. We analyze the force-dependence of the lifetimes of this intermediate state using the Arrhenius equation, where the separation free energy barrier can be reduced by the application of mechanical force to a molecule, which exponentially increases the transition rate. The rupture of molecular 


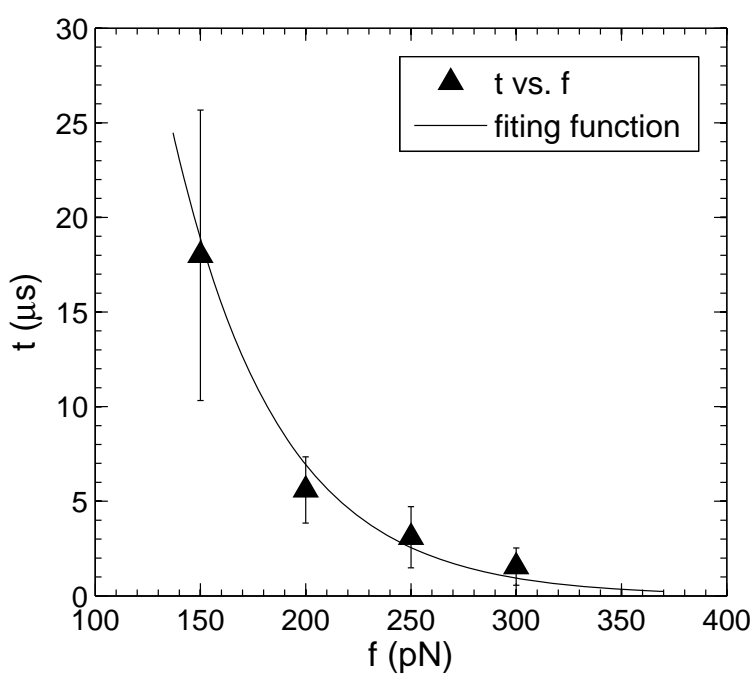

Figure 5: Force dependent lifetime of intermediate state $\alpha \beta_{1}$. Fitting function is $t=$ $379 * \exp (-0.02 * F)$. Standard deviation for each force value shown in error bars.

bonds under mechanical force was first studied by Bell (1978), who predicted that the life-time decreases exponentially with the stretching force which is defined as $t(F)=t_{0} \exp \left(-F \Delta x / k_{B} T\right)$, where $t_{0}$ is the lifetime without any clamping force and $\Delta x$ denotes the potential width. To study the forcedependence of the lifetimes of the $\alpha \beta_{1}$ intermediate state, we computed and analyzed the lifetimes for clamping forces ranging from 150 to $300 \mathrm{pN}$. The lifetime is estimated using Eq. 1 with $\nu=1 e^{9} \mathrm{~s}^{-1}$ (Dietz and Rief, 2004). In our SLME simulations as shown in Fig. 5, the average lifetime of the $\alpha \beta_{1}$ intermediate decreases with increasing force. By fitting the curve using Bell's formula, we obtain $t_{0}=379 \mu \mathrm{s}$ and $\Delta x / k_{B} T=0.02 \mathrm{pN}^{-1}$.

Finally, we comment on the average lifetimes of the three intermediate configurations that were observed in the $300 \mathrm{pN}$ unfolding simulations. In doing so, we find that the averaged lifetimes of $\alpha \beta_{1}, \alpha \beta_{1} \beta_{2} \beta_{3}$ and $\alpha \beta_{1} \beta_{2} \beta_{3} \beta_{11} \beta_{10}$ are 1.5, 1.8 and $6.89 \mu \mathrm{s}$, respectively. The intermediate states $\alpha \beta_{1}$ and $\alpha \beta_{1} \beta_{2} \beta_{3}$ have shorter lifetimes because the rupture of the hydrogen bonds is via tearing, with sequential breaking of bonds. However, for $\alpha \beta_{1} \beta_{2} \beta_{3} \beta_{11} \beta_{10}$, the pulling direction is perpendicular to the hydrogen bonds, resulting in a shearing mode of failure which is comparatively more difficult. These explanations are consistent with the explanation in the difference in lifetimes that 
can be obtained from the free energy profiles in Fig. 4. There, it is clear that $\alpha \beta_{1}$ and $\alpha \beta_{1} \beta_{2} \beta_{3}$ have smaller unfolding barriers and thus shorter unfolding time.

\section{Conclusions}

In conclusion, we have used the SLME simulation methodology to examine the force-induced unfolding of the protein GFP. Our key finding is the discovery of four total, and two new intermediate states that exist between 5 and $40 \mathrm{~nm}$ end-to-end distance, where the intermediate configurations, unfolding pathways, and lifetimes of the intermediate states is strongly forcedependent. The ability to unfold GFP to substantially longer end-to-end distances as compared to previous atomistic simulations is due to the timescale bridging nature enabled by the SLME methodology, and demonstrates the potential of this methodology for protein folding and unfolding problems.

\section{Acknowledgements}

HSP, WWT and PC acknowledge the support of NSF CMMI-1234183.

\section{References}

Bell, G.I., 1978. Models for the specific adhesion of cells to cells. Science 200, 618-627.

Berteotti, A., Barducci, A., Parrinello, M., 2011. Effect of urea on the $\beta$-hairpin conformational ensemble and protein denaturation mechanism. Journal of the American Chemical Society 133, 17200-17206.

Bustamante, C., Chemla, Y.R., Forde, N.R., Izhaky, D., 2004. Mechanical processes in biochemistry. Annual Review of Biochemistry 73, 705-748.

Cao, P., Li, M., Heugle, R.J., Park, H.S., Lin, X., 2012. A self-learning metabasin escape algorithm and the metabasin correlation length of supercooled liquids. Physical Review E 86, 016710.

Cao, P., Park, H.S., Lin, X., 2013. Strain-rate and temperature-driven transition in the shear transformation zone for two-dimensional amorphous solids. Physical Review E 88, 042404. 
Cao, P., Yoon, G., Tao, W., Eom, K., Park, H.S., 2015. The role of binding site on the mechanical unfolding mechanism of ubiquitin. Scientific Reports 5,8757 .

Caraglio, M., Imparato, A., Pelizzola, A., 2011. Direction-dependent mechanical unfolding and green fluorescent protein as a force sensor. Physical Review E 84, 021918.

Carrion-Vazquez, M., Li, H., Lu, H., Marszalek, P.E., Oberhauser, A.F., Fernandez, J.M., 2003. The mechanical stability of ubiquitin is linkage dependent. Nature Structural \& Molecular Biology 10, 738-743.

Dietz, H., Rief, M., 2004. Exploring the energy landscape of gfp by singlemolecule mechanical experiments. Proceedings of the National Academy of Sciences of the United States of America 101, 16192-16197.

Elber, R., 2005. Long-timescale simulation methods. Current opinion in structural biology 15, 151-156.

Eyal, E., Bahar, I., 2008. Toward a molecular understanding of the anisotropic response of proteins to external forces: insights from elastic network models. Biophysical journal 94, 3424-3435.

Fan, Y., Kushima, A., Yip, S., Yildiz, B., 2011. Mechanism of void nucleation and growth in bcc fe: atomistic simulations at experimental time scales. Physical review letters 106, 125501.

Fan, Y., Osetskiy, Y.N., Yip, S., Yildiz, B., 2013. Mapping strain rate dependence of dislocation-defect interactions by atomistic simulations. Proceedings of the National Academy of Sciences 110, 17756-17761.

Formoso, E., Limongelli, V., Parrinello, M., 2015. Energetics and structural characterization of the large-scale functional motion of the adenylate kinas. Scientific Reports 5, 8425.

Gromacs, 2014. http://www.gromacs.org/ .

Hanggi, P., Talkner, P., Borkovec, M., 1990. Reaction-rate theory: fifty years after kramers. Reviews of Modern Physics 62, 251-342. 
Hess, B., Kutzner, C., van der Spoel, D., Lindahl, E., 2008. GROMACS 4: algorithms for highly efficient, load-balanced, and scalable molecular simulation. Journal of Chemical Theory and Computation 4, 435-447.

Hub, J.S., de Groot, B.L., van der Spoel, D., 2010. g-wham a free weighted histogram analysis implementation including robust error and autocorrelation estimates. Journal of Chemical Theory and Computation 6, 37133720 .

Hyeon, C., Dima, R.I., Thirumalai, D., 2006. Pathways and kinetic barriers in mechanical unfolding and refolding of rna and proteins. Structure 14, $1633-1645$.

Kushima, A., Lin, X., Li, J., Eapen, J., Mauro, J.C., Qian, X., Diep, P., Yip, S., 2009. Computing the viscosity of supercooled liquids. The Journal of chemical physics 130, 224504.

Laio, A., Parrinello, M., 2002. Escaping free-energy minima. Proceedings of the National Academy of Science 99, 12562-12566.

Lau, T.T., Kushima, A., Yip, S., 2010. Atomistic simulation of creep in a nanocrystal. Physical review letters 104, 175501.

Li, J., Fernandez, J.M., Berne, B.J., 2010. Water's role in the force-induced unfolding of ubiquitin. Proceedings of the National Academy of Science 107, 19284-19289.

Marinelli, F., Pietrucci, F., Laio, A., Piana, S., 2009. A kinetic model of trpcage folding from multiple biased molecular dynamics simulations. PLOS Computational Biology 5, e1000452.

Mickler, M., Dima, R.I., Dietz, H., Hyeon, C., Thirumalai, D., Rief, M., 2007. Revealing the bifurcation in the unfolding pathways of gfp by using single-molecule experiments and simulations. Proceedings of the National Academy of Sciences 104, 20268-20273.

Neuman, K.C., Nagy, A., 2008. Single-molecule force spectroscopy: optical tweezers, magnetic tweezers and atomic force microscopy. Nature Methods $5,491-505$. 
Perez-Jimenez, R., Garcia-Manyes, S., Ainavarapu, S.R.K., Fernandez, J.M., 2006. Mechanical unfolding pathways of the enhanced yellow fluorescent protein revealed by single molecule force spectroscopy. Journal of Biological Chemistry 281, 40010-40014.

Popa, I., Fernández, J.M., Garcia-Manyes, S., 2011. Direct quantification of the attempt frequency determining the mechanical unfolding of ubiquitin protein. Journal of Biological Chemistry 286, 31072-31079.

Saeger, J., Hytönen, V.P., Klotzsch, E., Vogel, V., 2012. Gfp's mechanical intermediate states. PloS one 7, e46962.

Stirnemann, G., Giganti, D., Fernandez, J.M., Berne, B.J., 2013. Elasticity, structure, and relaxation of extended proteins under force. Proceedings of the National Academy of Science 110, 3847-3852.

Tao, W., Yoon, G., Cao, P., Eom, K., Park, H.S., 2015. $\beta$-sheet-like formation during the mechanical unfolding of prion protein. Journal of Chemical Physics 143, 125101.

Wales, D.J., 2002. Discrete path sampling. Molecular Physics 100, 32853305 .

Wales, D.J., 2004. Some further applications of discrete path sampling to cluster isomerization. Molecular Physics 102, 891-908.

Yan, X., Gouissem, A., Sharma, P., 2015. Atomistic insights into li-ion diffusion in amorphous silicon. Mechanics of Materials .

Yang, F., Moss, L.G., Phillips Jr, G.N., 1996. The molecular structure of green fluorescent protein. NATURE BIOTECHNOLOGY 14.

Zimmer, M., 2002. Green fluorescent protein (gfp): applications, structure, and related photophysical behavior. Chemical Reviews 102, 759-782. 